\title{
High Prevalence of Urinary Incontinence and Poor Knowledge of Pelvic Floor Exercises among Women in a Health District in KwaZulu-Natal, South Africa.
}

\begin{abstract}
Introduction: The purpose of the study was to determine the prevalence and health seeking behaviour of women with urinary incontinence and their knowledge of pelvic floor exercises, in a health district in KwaZulu-Natal.

Methods: An observational cross-sectional study design was used. Between September 2005 and November 2005, a questionnaire was administered to 99 women aged $21-76$ years at randomly selected from.

Results: The study found that the prevalence of the urinary incontinence was $35 \%$ (95\% Confidence Interval, 26 to 45). The most common type of incontinence was stress urinary incontinence, 63\% (95\% CI, 46 to 79). Of the 99 women, 32\% (95\% CI, 23 to 42) had heard of pelvic floor muscle exercises, while only $18 \%(95 \%$

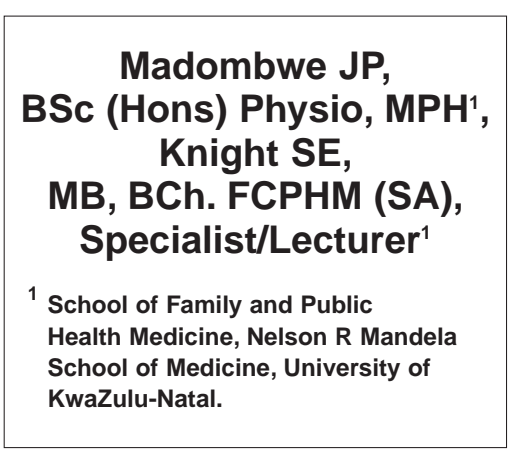
CI, $11-26)$ had actually carried out the intervention. Of the 35 women with urinary incontinence, $26 \%$ had sought professional help, the most common reason for seeking help being a worsening in condition.

Conclusion: The prevalence of urinary incontinence in KwaZulu-Natal is high, knowledge of urinary incontinence as a disease and its management, among both women and health service providers is poor.
\end{abstract}

KEY WORDS: URINARY INCONTINENCE, STRESS URINARY INCONTINENCE, URGE URINARY INCONTINENCE, MIXED URINARY INCONTINENCE, PREVALENCE, PELVIC FLOOR MUSCLE EXERCISES.

\section{ACKNOWLEDGEMENTS: \\ With special thanks to the South African Society of Physiotherapy for their assistance with funding this research.}

\section{INTRODUCTION}

Urinary Incontinence (UI) is a common but underreported problem globally amongst women, 1,2 with a prevalence between $27 \%$ and $42 \% .3,4,5,6,7$ There is very limited information on the prevalence and health burden of UI in South Africa and why so few women seek help for this manageable condition. In 1998, the South African Demographic and Health Survey measured the prevalence of Stress Urinary Incontinence (SUI) in South Africa for the first time, but only for women who had ever had children. ${ }^{2}$

In 1998, the World Health Organisation's first International Consultation on Incontinence classified UI as a disease and made recommendations on assessment, treatment and advocated to raise awareness about its symptoms and prevention. ${ }^{8,9}$ UI, an increasing public health issue in ageing populations, ${ }^{8}$ presents in three main types - Stress
Urinary Incontinence (SUI), Urge Urinary Incontinence (UUI) and Mixed Urinary Incontinence (MUI).

Both Pelvic Floor Muscle (PFM) exercises with and without biofeedback have been shown to significantly improve symptoms of UI. ${ }^{10}$ Randomised controlled trials and a Cochrane systematic review recommended that PFM exercises are an effective first-line, safe alternative treatment for all three types of incontinence. 1,8 Despite strong evidence for therapy being available, Australian women's knowledge, practices and intentions regarding correct PFM exercises in 2000 was poor and they required to be taught how to do the exercises correctly. ${ }^{11}$

UI is a social taboo and considered a normal consequence of childbirth and ageing. Sufferers remain silent about suffering with this disease due to embarrassment and a misconception that it cannot be treated. ${ }^{12}$ In 1998 , it was found that only $25 \%$ of incontinent Japanese women had consulted a doctor. $^{7}$ This was similar in Sweden where three out four incontinent women had never sought help as they felt that their incontinence was not a serious disease that needed professional care. ${ }^{13}$ This study aims to investigate the prevalence of UI, the health seeking behaviour and knowledge of pelvic floor exercises of women in a health district in KwaZulu-Natal.

\section{CORRESPONDENCE TO:}

Jackie Madombwe

Disability and Rehabilitation Coordinator Uthukela District Office

Private Bag X9958, Ladysmith 3370

Tel: (036) 631-2202

Cell: 076 112-4808

Email: jackie.madombwe@kznhealth.gov.za 


\section{METHODOLOGY}

This was an observational descriptive cross sectional study of 100 women randomly selected using dwelling units in the health district as a proxy sampling frame. Adult females over the age of 21 years formed the study population. Any women with mental retardation, other degenerative brain disease, spinal cord injuries, chronic urinary tract infection, or women on diuretic, anti-psychotic medication or opiates, and women over the age of 80 were excluded from the sample. Data on the number of children, number pregnancies, and method of delivery was collected but was not analysed as it was not the purpose of the study. Data was collected using a custom-designed questionnaire and administered by four trained interviewers, in the language of the respondent's preference. The questionnaire was validated by translators to identify any possible problems with wording. The questionnaire was then piloted by administering it to eight women in a gynaecologist's waiting room, to identify any further problems. As a result of this process some questions were modified to improve validity of the questionnaire.

To identify UI, the question "do you have any difficulty at all controlling urination?" was asked. There was a follow-up question on any past problems with controlling urination and all those who indicated a problem in the past 12 months were taken as having UI. The questionnaire included questions to determine type of incontinence, whether stress, urge or mixed, based on the definitions of the International Continence Society, ${ }^{14}$ a section on knowledge of PFM exercises and another section asking to who the patient with UI reported.

In order to reduce information bias, the four interviewers used to collect the data underwent intensive training on how to administer the questionnaire and the importance of asking the questions in a uniform manner using standard prompts where necessary.

Simple random sampling of dwelling units reduced selection bias. Where there was more than one eligible woman in a home, all were included in the sample. Using interviewer administered questionnaires overcame the problems of low response rate and dealing with the population with low literacy. Interviewing respondents in their language of preference reduced language bias. In most cases it was possible to match interviewers and respondents by race, reducing any possible cultural biases.

The Ladysmith community is a very small, urbanised community and most of the women use similar health facilities. This may present difficulties with generalising the results of this study to larger cities where there are a variety of health care options available. One possible confounder that was not accounted for was age, as UI is known to increase with age. 10

Data was collected between September 2005 and November 2005. The study was explained to the women and permission to interview them was obtained by written consent. Each woman was left with a leaflet that explained UI and gave basic instructions on how to perform pelvic floor exercises. All statistical computations were done by a statistician, using the SAS version 9 programme.

Ethical approval was sought and full ethical approval was obtained from the Biomedical Research Ethics Committee and the Postgraduate Education Committee of the University of KwaZulu-Natal, School of Medicine. Permission was also sought and granted from the Ladysmith Municipality.

\section{RESULTS}

Out of 100 sampled households, in two households the women refused to be interviewed and in another two, the women were unavailable. In six households there were two women in residence. A total of 102 women were interviewed but three women were excluded because one was pregnant, one was 83 years old and one was on diuretics which brought the number of women included in the study to 99.

\section{Prevalence}

Of the 99 women, $35.4 \%$ (35) (95\% CI, $25.9-44.8)$ reported UI. The majority of the 35 incontinent women $(62.9 \%$; 95\% CI, 46.5 - 79.2) had SUI (23), $31.4 \%$ (95\% CI, 15.7 - 47.1) had MUI (11), while only $2.9 \%$ (95\% CI, -2.8 8.5) had UUI (1).

The total prevalence of SUI was $23.2 \%$ (23) (95\% CI, 14.8 - 31.7), MUI was $11.1 \%$ (11) (95\% CI, $4.8-17.4)$ and UUI was $1.0 \%$ (1) $(95 \% \mathrm{CI},-1.0-3.0)$, $\mathrm{n}=99$. (Fig 1).

Figure 1: Prevalence (\%) of types UI in women in Ladysmith, 2005 (N=99)

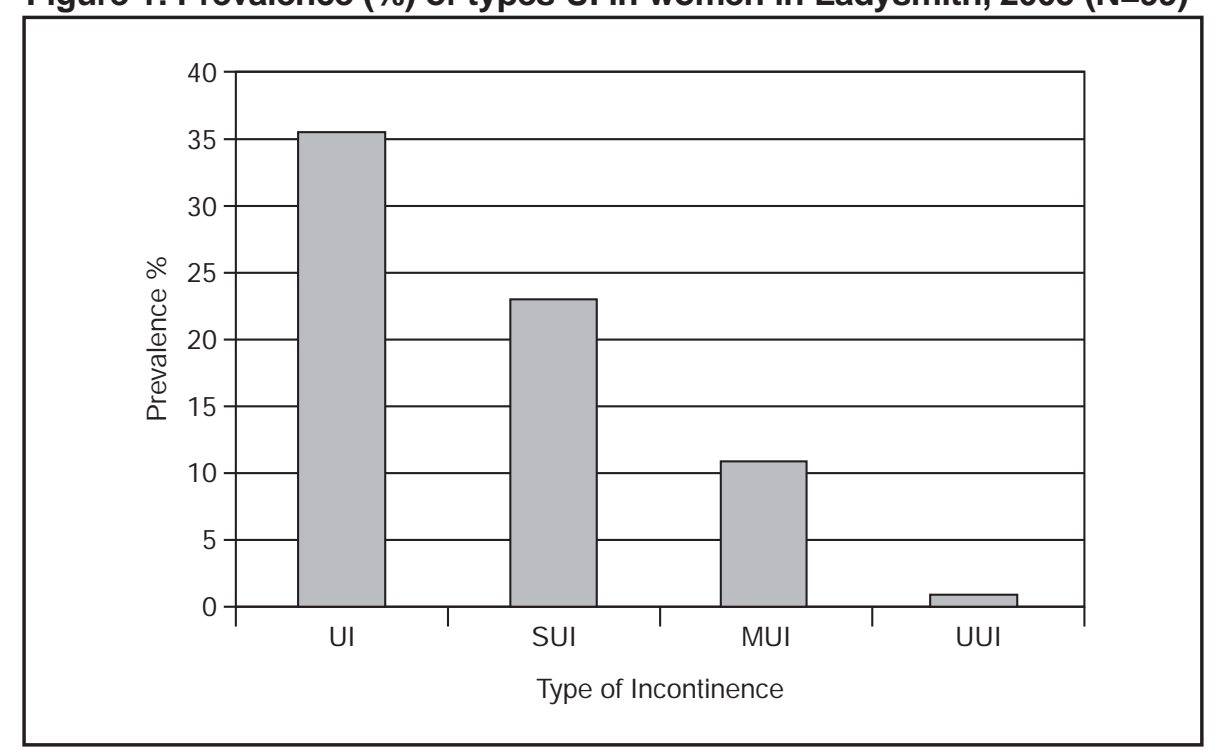

2 Women who actually had knowledge of how to perform the PFM exercises, whether it was from instructions in a magazine or formal instructions from a professional. 
The mean age of the total sample was 43.7 years $(95 \% \mathrm{CI}, 40.3-45.8)$ and the median age was 43.0 years. Using the Shapiro-Wilk Test $(p=0.22)$ to test for normality, the ages of women in the sample were assessed to be normally distributed. However, the size of age groups varied. The 31-40 year age group had the most women (29) with only two women in the sample in the 71-80 year age group.

The largest proportion of cases was in the $41-50$ year age group, $42.9 \%$ (15) (95\% CI, $24.2-61.6)$. The mean age of the women without UI was 41.6 years (95\% CI, 39.1 - 45.9), while the mean age of the women with UI was 45.5 years $(95 \% \mathrm{CI}, 41.7-49.4)$. There was no significant difference between the means of the two groups ( $\mathrm{t}$ test $=-1.26$, $\mathrm{p}=0.05)$.

\section{Figure 2: Age distribution of study sample ( $\mathrm{N}=99)$}

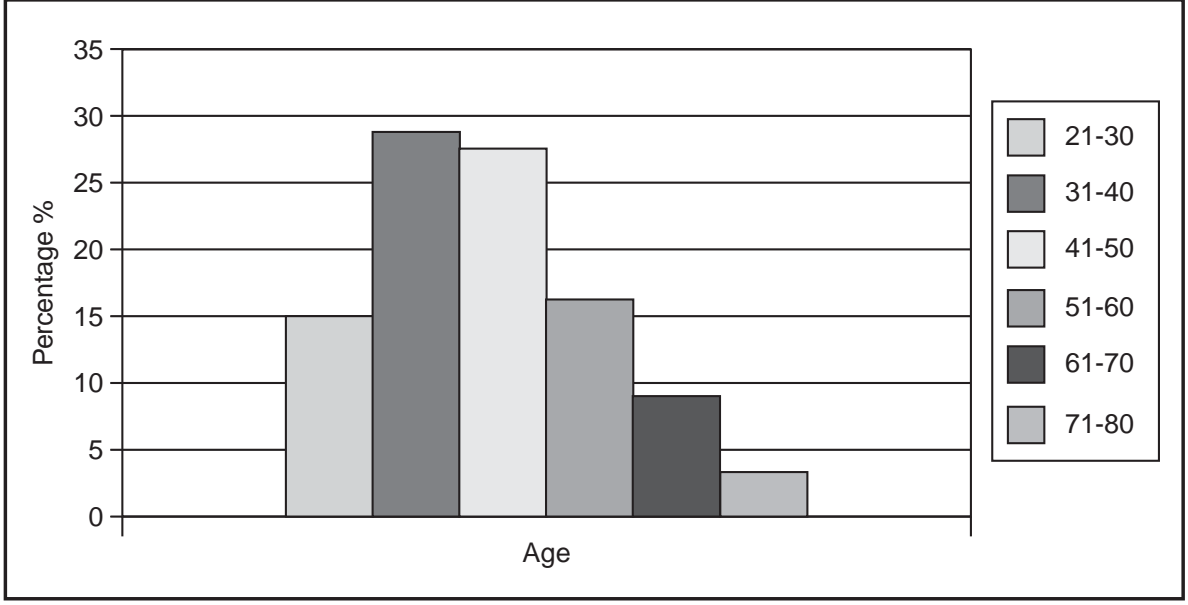

Table 1: Reasons women with Ul gave for seeking help, $n=9$

\begin{tabular}{|l|l|}
\hline Reasons for seeking help & \\
\hline Urine leakage worsened & 6 \\
\hline Leakage is shameful and embarrassing & 4 \\
\hline Leakage caused decreased physical activity & 1 \\
\hline Did not want problem to worsen & 1 \\
\hline
\end{tabular}

(More than one reason could be given)

Table 2: Reasons women with UI gave for not seeking help, $\mathrm{n}=\mathbf{2 6}$

\begin{tabular}{|l|l|}
\hline Reasons for not seeking help & \\
\hline Leakage is part of normal ageing & 4 \\
\hline Leakage is normal after childbirth & 7 \\
\hline Leakage is only a small problem & 20 \\
\hline Did not know what types of help are available & 1 \\
\hline
\end{tabular}

(More than one reason could be given) ing help was that it was considered a small problem. (Tables 1 and 2). Two of the women who sought help did not attend their follow-up visits. For one of the women, it was because she was not happy with the service she received at the clinic and she did not know what other help was available. Further more, she was not financially able to seek any further help, although she felt that her condition was worsening.

\section{DISCUSSION}

UI was found to be common, with a prevalence that may even be higher than the reported $35.4 \%$ (35), as some women may have been too embarrassed to admit to such a condition. However, the $35.4 \%$ prevalence is consistent with other previous studies. $4,5,7$ A higher prevalence of $40.7 \%$ in 2004 and $42 \%$ in 2005 was found among Chinese and Rwandan women respectively. ${ }^{15,3}$

SUI has been said to be the most common type of UI and is commonly associated with childbirth and pregnancy. ${ }^{1}$ In this study, SUI was found to be the most common of the three types of UI, followed by MUI and UUI. Luna et al found that of the women with UI, $80 \%$ had SUI, $14 \%$ had MUI and $4 \%$ had UUI. ${ }^{7}$

Out of the total sample of 99 women, this study found a $23.2 \%$ (23) prevalence of SUI. This is more than double the $10 \%$ prevalence of SUI reported by the 1998 SADHS. $^{2}$ It may be that the prevalence has increased, as was found by Wong et al among Hong Kong Chinese women, where prevalence of SUI had increased from $21 \%$ in 1996 to $40.7 \%$ in 2005.15 The difference in prevalence may also be due to the 1998 SADHS not having been an incontinence specific survey. The question on incontinence was one among many other questions dealing with other health issues and the question may not have been stressed adequately, resulting in respondents not considering it as important.

Only $32.3 \%$ (32) of the 99 women interviewed had heard of PFM exercises, and $18.1 \%$ (18) had actually done the exercises. This is a very low prevalence out of a sample of largely parous women, who would be expected to have actually been taught the PFM exercises 
at their antenatal visits. Chiarelli et al. in 2003, found that out of a sample of 720 women, $95.7 \%$ had heard of PFM exercises but their study concluded that despite good knowledge of PFM exercises, few women actually did them. ${ }^{11}$

What was most disturbing in this study was that even women with UI were not being taught PFM exercises as a first line of treatment. The Cochrane review on effectiveness of PFM exercises concluded that they were an effective treatment method of UI. ${ }^{1}$ The evidence shows that they are not been taught or done. There appeared to be a disturbing lack of knowledge among health practitioners in Ladysmith as to UI and its assessment and treatment.

If women could be taught PFM exercises to prevent UI, long term morbidity associated with pregnancy and childbirth would be reduced and the problem could be prevented from worsening and resulting in a need for surgical intervention. It has been shown that surgical intervention is not the ultimate solution to UI. In the current study one woman with UI had already undergone three surgical procedures, without attaining total continence. If women could be treated with exercise, it would certainly lessen the costs incurred due to surgery and the purchase of incontinence products such as protective pads.

The study found that only $25.7 \%$ (9) of the women with UI had sought professional help. Luna et al. reported that $25 \%$ of the women with UI in their study sought medical help and the most common reason for seeking help was worsening of the problem. ${ }^{7}$ Hunskaar et al. found a consultation rate of $30 \% .^{4}$ Wong et al. found that $61.2 \%$ thought that leakage of urine was a normal aging process and over $90 \%$ of the women felt that education on incontinence was not enough. ${ }^{15}$ There appears to be low consultation rates worldwide and the reasons for this are the same among women of different cultures and backgrounds. Other researchers felt that primary healthcare providers lacked confidence in the management of UI and this led to under-treatment in those patients seeking help. ${ }^{4}$

The size of this study was a major limitation. It was difficult to obtain meaningful estimates of age-specific prevalence because the sample sizes were too small and differed greatly across the age groups. Unfortunately the study did not obtain information on the length of stay of each respondent in the area chosen for the study. This information would have given a clearer picture on whether the problems or lack of knowledge were due to deficiencies in services in this particular health district or elsewhere.

\section{CONCLUSION AND RECOMMENDATIONS}

UI is very common among adult women in this health district in KwaZulu-Natal, South Africa, with a prevalence of $35.4 \%$. The women most affected were found to be those in the $41-50$ year age group. The prevalence of UI among South African women appears to have increased over the past seven years, if the prevalence of SUI is to be used as an indicator of this increase.

This study has also shown that knowledge of PFM exercises is poor and knowledge concerning management of UI among the health service providers needs to be improved. It is strongly recommended that primary health care providers familiarize themselves with incontinence and its management. The study also highlighted the lack of awareness among women, of UI as a disease. There is a clear need for public health programmes to address the problem of UI in the community by providing education on strategies for prevention and treatment, and to make women more aware of it as a medical condition that can be treated. Larger studies need to be done in other communities in South Africa and it will also be beneficial to determine the effect of UI on quality of life of women in South Africa.

\section{REFERENCES}

1. Hay-Smith EJC, Bf K, Berghmans LCM, Hendricks HJM, de Bie RA, van Waalwijk van Doorn ESC. 2001. Pelvic floor muscle training for urinary incontinence in women. Cochrane review. The Cochrane Library 2004, Issue 2. John Wiley and Sons Ltd.

2. Department of Health. Medical Research Council. 1998. Maternal and Child Health. In: South African Demographic and Health Survey Preliminary Report. Chap. 7. 119-120. Macro International Inc.
3. Gashugi P, Louw Q. 2005. Prevalence and risk factors of urinary incontinence among adult Rwandan women. S Afr J Physiotherapy 61(4): $6-14$.

4. Hunskaar S, Lose G, Sykes D, Voss S. 2004. The prevalence of urinary incontinence in women in four European countries. BJU International 93: $324-330$.

5. Perry S, Shaw C, Assassa P, Dallosso H, Williams K, Brittain KR, et al. 2000. An epidemiological study to establish the prevalence of urinary symptoms and felt need in the community: the Leicestershire MRC Incontinence Study. J Public Health Med 22(3): 427 - 434.

6. Peyrat L, Haillot O, Bruyere F, Boutin JM, Bertrand P, Lanson Y. 2002. Prevalence and risk factors of urinary incontinence in young and middle-aged women. BJU Int 89: 61 - 66 .

7. Luna MTC, Hirakawa T, Nakano H. 2000. Urinary incontinence in Women seen in the obstetrics and gynaecology clinic. Int Urogynecol J Pelvic Floor Dysfunct 11: $277-281$

8. Abrams P, Lowry SK, Wein AJ, Bump R, Denis L, Kalache A, et al. 2000. Assessment and treatment of urinary incontinence. Consensus. Lancet 355: $2153-2158$.

9. WHO. 1998. World Health Organization calls first international consultation on incontinence. Press Release WHO/49. Available from www.who.int Accessed on 23/11/2005.

10. Aksac B, Aki S, Karan A, Yalcin O, Isikoglu M, Eskiyurt N. 2003. Biofeedback and pelvic floor exercises for the rehabilitation of urinary stress incontinence. Gynecol Obstet Invest 56: 23-27

11. Chiarelli P, Murphy B, Cockburn J. 2003. Women's knowledge, practises, and intentions regarding correct pelvic floor exercises. Neurourol Urodyn 22: 246 - 249.

12. Morris K. 1999. Tackling the taboo of urinary incontinence. Lancet 353: 128

13. Hagglund D, Walker-Engstrom M, Larsson G, Leppert J. 2003. Reasons why women with long-term urinary incontinence do not seek professional help: a cross-sectional population-based cohort study. Int Urogynecol J Pelvic Floor Dysfunct 14: 296 - 304

14. Sand PK, Dmochowski R. 2002. Analysis of the standardisation of terminology of lower urinary tract dysfunction: Report from the standardisation sub-committee of the international continence society. Neurourol Urodyn 21: $167-78$.

15. Wong T, Mak HLJ. Cheon WC, Pang MW, Yip SK. 2005. Urinary incontinence in Hong Kong Chinese women: A prevalence, quality of life, and knowledge study. Presented at International Continence Society Conference 2005 Montreal, Canada. Category: Epidemiology and Outcomes Research. Available from www.icsoffice.org Accessed on 10/01/2006. 